Rev, Elev. Med. Vét. Poys trop., 1967, 20, I (105-108).

\title{
Caballerocotyla klawei Stunkard 1962, Monogène parasite des sacs nasaux du thon albacore de l'Océan Atlantique
}

\author{
par J. BUSSIERAS ef J.-F. ALDRIN \\ (Laboratoire de Parasitologie, Ecole Nationale Vétérinaire de Lyon, \\ Laboratoire de Contrôle des Prodults de la Pêche, Abidjan, Côte d'Ivoire).

\section{RÉSUME} \\ Les auteurs étudient les Cabollerocotyla klowei STUNKARD 1962 récoltés dans \\ les cavités nasales des thons Thunnus albacares pêchés dans l'Océan Atlantique, et \\ les comparent à la description des parasites analogues récoltés dans l'Océan \\ Pocifique.
}

La présence de Monogènes du type «Tristome 》 (sous-famille des Capsalinoe JOHNSTON 1929) dans les cavités nasales des thons albacores Thunnus albacares Bonnaterre pêchés dans l'Atlantique, est connue depuis plusieurs années. Elle fut signalée par ROSSIGNOL et REPELIN (1962), puis vérifiée par l'un de nous (ALDRIN 1965). II est curieux de constater que ces divers auteurs ont retrouvé des Tristomes dans les cavités nasales de tous les thons albacores examinés, et d'eux seuls.

Les Thunnus albacares pêchés dans l'Océan Pacifique (appelés Thunnus macropterus par ceux qui considèrent qu'il s'agit peut-être d'une espèce différente) hébergent également des Tristomes dans leurs cavités nasales. Le fait a été noté par KLAWE (rapporté par M. B. SCHAEFER et Coll. 1962) qui a admis, sans doute un peu rapidement, que ces parasıtes appartiennent à l'espèce Capsala martinieri. Mais une vérıtable étude parasıtologique des spécimens récoltés dans l'Océan Pacifique fut entreprise par H.W. STUNKARD, qui montra qu'il s'agissait d'une espèce nouvelle, Caballerocotylo klawei STUNKARD 1962 ; à la fin de son article, STUNKARD signale en quelques mots que $E$. W. PRICE a pu comparer des exemplaires.de ce C. klowei à d'autres provenant des thons albacores des côtes d'Afrique, ta conclusion de E. W. PRICE étant que les albacores de l'Atlantique et du Pacifique hébergent bien le même C. klawei.

II nous a cependant paru bon de reprendre l'examen des Tristomes provenant de l'Atlantique (au large des côtes africaines entre Dakar et Abidjan) pour les comparer à ceux de l'Océan Pacifique décrits par STUNKARD.

\section{ÉTUDE DES CABALLEROCOTYLA KLAWEI RÉCOLTÉS DANS L'OCÉAN ATLANTIQUE}

II ne semble pas nécessaire de décrire de façon détaillée le parasite, car cette description reprendrait en très grande partie celle de STUNKARD.

Nous ne retiendrons done que les points particuliers qui ont permıs d'observer quelques différences avec la description originale.

a) Dimensıons des parasites fixés.

En général, les dimensions que nous avons obtenues sur 5 spécimens fixés, colorés ef montés in toto (fig. 1) sont très largement supérieures à celles du parasite du Pacifique.

Toutes les dimensıons seront exprimées en millimètres. 


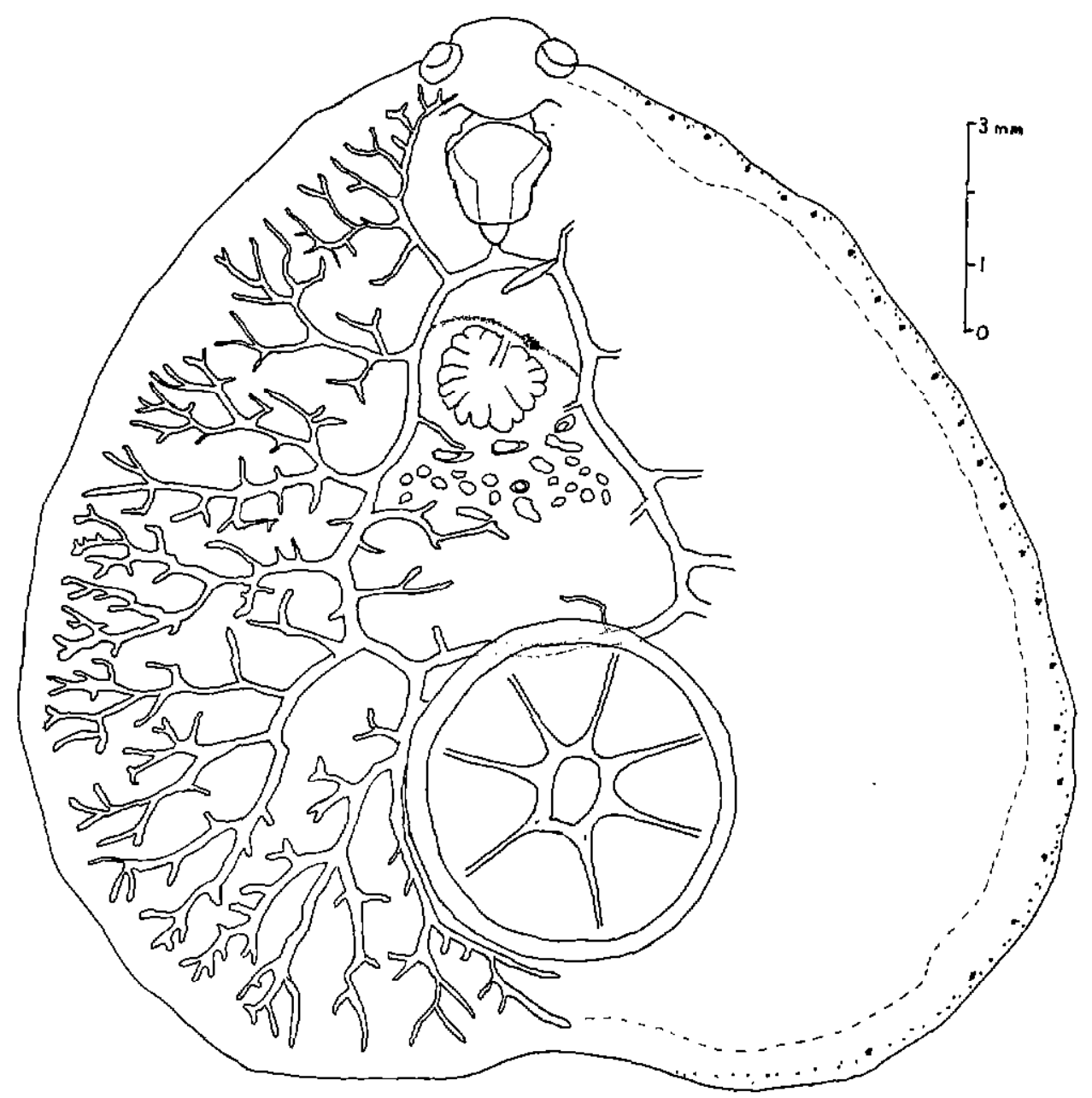

Fig. 1. - Caballerocotyla klawei, vue ventrale; à gauche sont représentées les ramifications des cæcums digestisf, à droite les épines de la face dorsale et, en pointıllé, la limite du territoire occupé par les glandes vitellogènes.

Longueur totale..... Largeur totale ......

Diamètre transversal des prohapteurs ... Distance entre les prohapteurs.......... Diamètre de l'opisthapteur (membrane périphérique comprise) ............ Longueur des ancres de l'opisthapteur...

$\left.\begin{array}{cc}\begin{array}{c}\text { C. Klawei } \\ \text { de I'Océan } \\ \text { Pacifique }\end{array} & \begin{array}{c}\text { C. klawei } \\ \text { de l'Océan } \\ \text { Atlantique }\end{array} \\ \text { (valeurs données } \\ \text { par STUNKARD) }\end{array}\right)$

b) Appareil génital (fig. 2).

La description de STUNKARD est valable pour les parasites de I'Océan Atlantique, à quelques détails près :

- le réceptacle séminal est réuni par un conduit, non à l'oviducte, mais au réservoir vitellin; une telle disposition avait d'ailleurs déjà été observée par GOTO (1895) sur toutes les espèces de Tristomes étudiées par lui ,

- on peut observer dans chaque ver, au voisinage des testicules et de l'ovaire, la présence d'environ 12 «glandes de Goto», selon la terminologie proposée par MESERVE et utilisée par BAER et EUZET (1961). Chez C. klawei, elles 


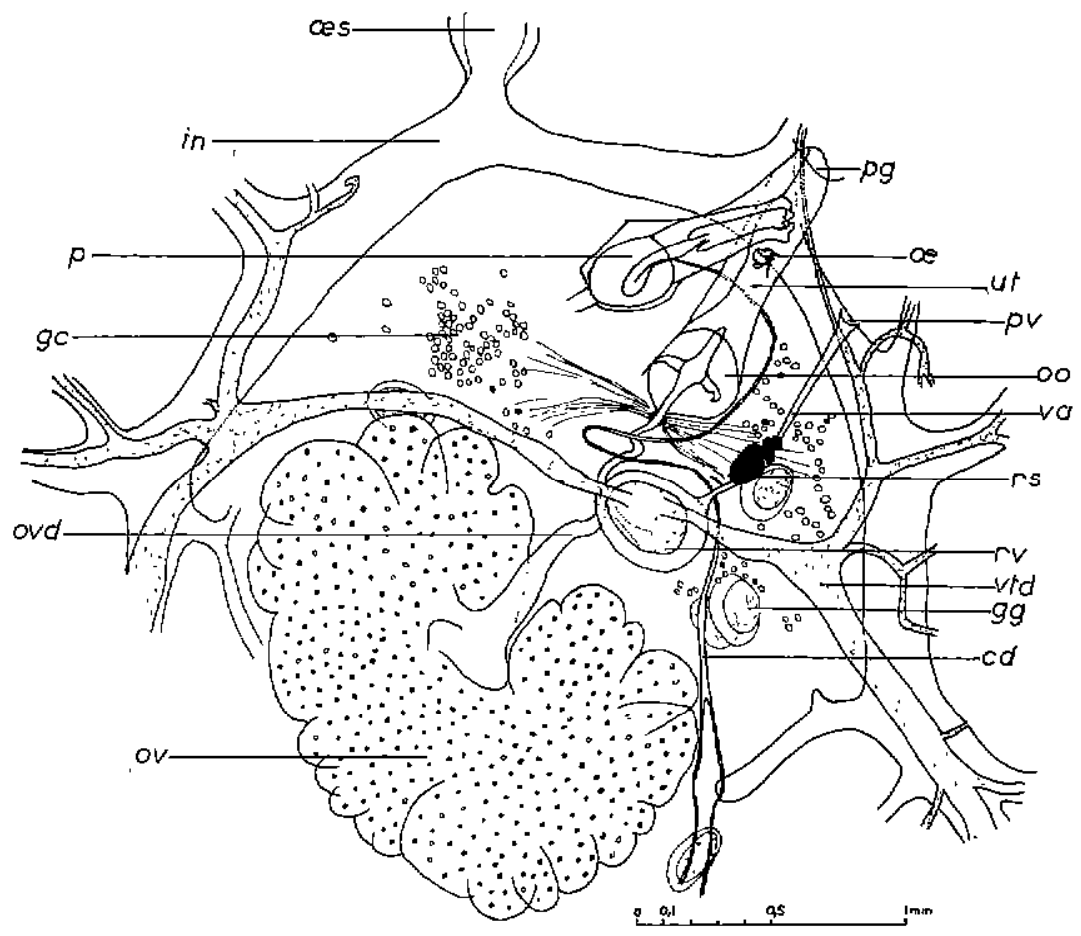

Fig. 2. - Caballerocotyla klowel, appareil génital (vue partielle, face ventrale) cd, canal déférent; gc, glandes coquillières ; gg, glonde de Goto ; in, intestin ; $œ$, œuf ; œs, œsophage ; oo, ootype ; ov, ovaire ; ovd, oviducte ; p. pénıs i pg, pore génital; $p v$, pore vaginal ; $r s$, réceptacle séminal ; $r v$, réservoir vitellin ; ut, utérus ; va, vagin ; vtd, vifelloducte.

se présentent comme des masses syncytiales de forme allongée, dont le grand axe mesure de 0,175 à $0,228 \mathrm{~mm}$, le petit axe de 0,160 à $0,175 \mathrm{~mm}$ chacune renferme 4 d̀ 12 énormes noyaux très régulièrement sphériques, de 0,023 à $0,027 \mathrm{~mm}$ de diamètre; chaque noyau contient habituellement 2 nucléoles;

- sur les 5 exemplaires montés in toto, 3 contenaient un ceuf, l'un situé dans l'utérus (fig. 2) les deux autres dans l'ootype ;

- enfin, nous avons retrouvé un ceríain nombre de différences avec les données numériques établies par STUNKARD :

\begin{tabular}{|c|c|c|}
\hline & $\begin{array}{l}\text { C. klawel } \\
\text { de l'Océan } \\
\text { Pacifique } \\
-\end{array}$ & $\begin{array}{l}\text { C. Klower } \\
\text { de l'Océan } \\
\text { Atlantique } \\
-\end{array}$ \\
\hline $\begin{array}{l}\text { Nombre de testicules..... } \\
\text { Diamètre des testicules ... } \\
\text { Longueur du pénis....... } \\
\text { Diamètre maximal du pé. }\end{array}$ & $\begin{array}{c}24-28 \\
0,12-0,18\end{array}$ & $\begin{array}{c}24-34 \\
0,16-0,27 \\
0,71-0,82\end{array}$ \\
\hline $\begin{array}{c}\text { Diamètre maximal du pé- } \\
\text { nis } \ldots \ldots \ldots \ldots \ldots \ldots \ldots \ldots . .\end{array}$ & non précisés & $0,17-0,24$ \\
\hline $\begin{array}{r}\text { Ovaire : grand axe } . . . . \\
\text { petit axe ....... }\end{array}$ & $\begin{array}{l}0,85-1,25 \\
0,80-0,90\end{array}$ & $\begin{array}{l}1,53-2,30 \\
1,27-1,64\end{array}$ \\
\hline
\end{tabular}

c) Epines du bord de la face dorsale.

Ces épines, comparables à celles observées sur les parasites de l'Océan Pacifique, sont souvent becucoup plus nombreuses, puisqu'on peut en compter, sur un seul côté de ver, jusqu'à 332 pour la rangée marginale de petites épines, et 24 pour la rangée d'épines plus volumineuses (alors que STUNKARD n'en avalt compté respectivement que 130 et 20 ).

De plus, même après coloration au carmin acétıque, il n'a pas été possible d'observer de zone claire entourant chaque épine.

\section{DISCUSSION}

Les légères différences observées entre les deux types de parasites ne nous paraissent pas suffisantes pour consıdérer qu'il s'agit de deux variétés distinctes. Seule l'étude comparée de nombreux spécimens des deux provenances permettra de dire s'il existe réellement une variété atlantica particulière. 
Remarquons par ailleurs que l'introduction de cette espèce dans le genre Caballerocotyla PRICE 1960 peut prêter à discussion, car :

- le pharynx ne présente qu'une ébcuche d'étranglement vers la moitié de sa longueur, ce qui lui donne un aspect bien différent de celui du pharynx des autres Caballerocotyla;

- le rapport : diamètre d'un prohapteur/ diamètre de l'opisthapteur, qui est généralement compris entre $1 / 7$ et $1 / 6$, ne correspond pas à la définition du genre donnée par PRICE (rapporf compris entre $1 / 3$ et $1 / 2$ ) ;
- le caractère « ouvert » de l'opisthapteur, selon la terminologie proposée par GUIART (1938) et adoptée par PRICE (1960), est peu marqué, notamment sur les exemplaires fixés ; ce fait, qui avait déjà été noté auparavant sur d'autres espèces, rend ce caractère difficilement utilisable en systématique.

Enfin, il sera intéressant ultérieurement de savoir si le taux d'infestation des albacores de l'Océan Pacifique est de l'ordre de 100 p. 100 , comme c'est le cas chez les albacores de l'Océan Atlantique.

\title{
SUMMARY
}

Caballerocotyla klawei STUNKARD 1962 monogenous parasite of the nasal cavities of Thunnus albocares of the Atlantic Ocean

Caballerocotyla klawei STUNKARD 1962 found in the nostril of tunny fishes (Thunnus albacores) from the Atlanilic Ocean has been studied and compared with the description of similar parasites gathered in the Pacific Ocean.

\section{RESUMEN}

Caballerocolyla klawei STUNKARD 1962, monogene parásito de la cavidaó nasal de Thunnus olbacares del Oceano Atlántico

\begin{abstract}
Se estudian los Cobalierocotyla klawei STUNKARD 1962 recogidos en las cavidades nasales de los atunes Thunnus abocares pescados en el Oceano Atlántico; se comparan los dichos parásitos con la descripción de los análogos recogidos en el Oceano Pacifico.
\end{abstract}

\section{BIBLIOGRAPHIE}

ALDRIN (J. F.). - «Observations sur la technologie ef l'inspection des Thonidés ». Rec. Méd. Vét. 1965. 141 (8) 679-713.

BAER (J.) et EUZET (L.). - « Classe des Monogènes $\gg$, in Traité de Zoologie de P. P. Grassé, IV, fasc. 1, 1961, 243-325.

GOTO (S.). - « Studies on ectoparasitic Trematodes of Japan ». J. Coll. Sc., 1895, 8, $1-273$.

GUIART (J.). - «Trématodes parasites provenant des Campagnes scientifiques du Prince Albert ler de Monaco (1886-1912) 》. Résultats des Camp. scient. Albert ler Monaco, 1938, 100, 7.

PRICE (E.W.). — « The giant marlin, Makiara marlina Jordan and Evermann, a new host for Capsala pricei Hidalgo 1959, with a review of the subfamily Capsalinae ». Libr. Hom. al Dr Caballero y Caballero, 1960, 237-244.

ROSSIGNOL (M.) et REPELIN (R.). - «Présence d'un Trématode parasite des sacs nasaux chez $N$. albacora (Lowe) ». Travaux Centre Océanogr. Pointe Noire, 1962, 2, 177 et 288 .

SCHAEFER (M. B.), BROADHEAD (G. C.) et ORANGE (C. J.). - « Synopsis on the biology of yellowfin tuna Thunnus albacores Bonnaterre 1788 (Pacific Ocean) 》. InterAmerican Tropical Tuna Commission, La Jolla, Californie. 1962.

STUNKARD (H.W.) - « Caballerocotyla klawei sp. n., a monogenetic trematode from the nasal capsule of Neothunnus mocropterus ». J. of Poras., 1962, 48 (6), 883-890. 